\title{
Mantle convection behavior with segregation in the core-mantle boundary
}

\author{
Günter Nauheimer ${ }^{1}$ Anatoly S. Fradkov ${ }^{2}$ Horst J. Neugebauer ${ }^{1}$
}

\begin{abstract}
Dynamical effects at the core-mantle boundary have so far been modeled in the frame of thermal as well as double-diffusive convection, yet accumulating evidence supports the idea that the mantle material looses part of its heavy component at the CMB due to combined chemical and physical processes. The segregation of the heavy $\mathrm{FeO}$ component from the lowermost mantle to the top of the core occurs inside the lower thermal boundary layer and leads to a new kind of boundary layer instability. A mathematical description of mantle convection including segregation of $\mathrm{FeO}$ into the core is developed and the relevant model parameters are estimated. The numerical investigation of the flow structure shows the forming and up-welling of thermo-compositionally driven plumes, as well as downwelling of pure thermally driven plumes even for conditions where the simple thermal convection case has a steady behavior. Periods of quasi-stable plume activity interchange with periods of chaotic reorganization of the flow structure connected with strongly time dependent Nusselt.
\end{abstract}

\section{Introduction}

Theoretical investigation on the solubility of $\mathrm{FeO}$ in molten Fe [Ringwood, 1984] as well as laboratory experiments on mantle silicates in contact with liquid iron [Knittle and Jeanloz, 1989], suggest that the core-fluid interacts chemically with the mantle silicates over geologic time [Artyushkov, 1972]. The mantle silicates are depleted in $\mathrm{FeO}$ by this process and a light residue consisting of iron-depleted enstatite and stishovite is left, which possibly rises in the form of plumes due to its enhanced buoyancy.

Theoretical investigation of McCammon et al. [1983] on the system $\mathrm{Fe}-\mathrm{FeO}-\mathrm{MgO}$ suggest that $\mathrm{FeO}$ may gradually ex-solve from ( $\mathrm{Mg}, \mathrm{Fe}) \mathrm{O}$ to become a separate phase in CMB. The melting point measurement of $\mathrm{FeO}$ from Boehler [1992] suggests that free $\mathrm{FeO}$ should be liquid and mobile inside the lower thermal boundary layer (CMB). Because $\mathrm{FeO}$ is the dense mantle component and because it is miscible with $\mathrm{Fe}$ [Ringwood and Hibberson, 1991], FeO moves down to the core mantle interface and enters the core.

The theory of terrestrial heat flow shows that most of the Earth's heat loss comes from non-radiogenic sources

\footnotetext{
${ }^{1}$ Geodydamik-Physik der Lithosphäre, Universität Bonn, Germany

${ }^{2}$ Earth \& Planetary Sciences, McGill University, Montreal, Canada
}

Copyright 1996 by the American Geophysical Union.

Paper number 96GL02062

0094-8534/96/96GL-02062\$05.00
[Oxburgh and $O$ 'Nions, 1987]. To explain this, secular cooling and the release of gravitational potential energy due to growths of the core has been adopted [Vacquier, 1991].

The proposed loss of a heavy mantle component at the core/mantle interface is a density unstable situation leading to a new kind of boundary layer instability, whose influence on convective mantle dynamics is studied numerically.

\section{Equations}

We use a two component, incompressible, infinite Prandtl number model-fluid in a 2D Cartesian geometry for investigating mantle convection behavior with segregation in CMB. The heavy component (described by a volume concentration $C$ ) represents $\mathrm{FeO}$ and other siderophile elements that take part in the segregation process. The bulk fluid represents the residual silicate mantle. The density of the mixture linearly depends upon temperature and composition according to $\varrho_{m}=\varrho_{s}\left(1-\alpha\left(T-T_{0}\right)+\beta C\right), \alpha$ being the thermal expansivity and $\beta=\frac{\varrho_{f}-\varrho_{s}}{\varrho_{s}}$ describing density changes due to composition ( $e_{f}$ is density of pure $\mathrm{FeO}, \varrho_{s}$ is the density of pure silicate).

In an effective medium approach, valid on geologic time and length scales, the segregation of $\mathrm{FeO}$ and other siderophiles is modeled by a phenomenological transport coefficient, the segregation velocity $v_{s}$, parameterizing a complex physical process. The segregation process can possibly occur within different hydrodynamical modes during the Earth's history [Stevenson, 1991], i.e. the segregation velocity naturally depends upon the temperature, pressure and chemical environment of the core/mantle reflecting for example kinetic limitations of the $\mathrm{FeO}-\mathrm{MgO}$ ex-solution. For ease of discussion, $v_{s}$ is only depth dependent here. The conservation law for iron and the other siderophiles including segregation is thus given by:

$$
\frac{\partial \varrho_{f} C}{\partial t}+\left\{\begin{array}{cl}
\nabla \cdot\left(\varrho_{f} C \vec{v}\right) & : \text { above D" } \\
\nabla \cdot\left(\varrho_{f} C\left(\vec{v}+v_{s} \frac{\vec{g}}{|g|}\right)\right) & : \text { inside D" }
\end{array}\right\}=0
$$

With $\vec{v}$ convective velocity and $v_{s}$ segregation velocity. Because the diffusive transport of $\mathrm{FeO}$ is much smaller than the advective one, we have a priory neglected the diffusive transport of the $\mathrm{FeO}$ component.

The above mentioned approximations and the model approach for the transport of $\mathrm{FeO}$ leads to the following system of non-dimensional equations [Trubitsyn and Kharybin, 1987; Fradkov et al., 1995]

$$
\begin{gathered}
\Delta \Delta=R_{T}\left(\frac{\partial \tilde{T}}{\partial x}-R_{p} \frac{\partial \tilde{C}}{\partial x}\right) \\
\frac{\partial \tilde{T}}{\partial t}+\nabla \cdot \tilde{T} \vec{v}=\Delta \tilde{T}
\end{gathered}
$$




$$
\begin{aligned}
\frac{\partial \tilde{C}}{\partial t}+\nabla \cdot \tilde{C} \vec{v} & =\left\{\begin{array}{cl}
0 & : \text { above D" } \\
-\frac{\partial a \tilde{C}}{\partial z} & : \text { inside D" }
\end{array}\right. \\
R_{T}=\frac{\alpha \Delta T g L^{3}}{\nu \kappa}, R_{p} & =\frac{\beta C_{o}}{\alpha \Delta T}, a=\frac{v_{s} L}{\kappa}
\end{aligned}
$$

where $\psi$ is the stream function; $\tilde{T}$ is the dimensionless temperature; $\tilde{C}$ is the dimensionless volume concentration of FeO; $R_{T}$ is the Rayleigh number; $R_{p}$ is the density number; $a$ is the segregation parameter; $\Delta T$ is the temperature drop; $g$ is the acceleration of gravity; $L$ is the mantle depth; $\kappa$ is the thermal diffusivity; $\nu$ is the viscosity; $C_{o}$ is the initial volume content of $\mathrm{FeO}$.

Because initial conditions are very difficult to define on physical grounds, we used a homogeneous FeOdistribution and a steady thermal convection state from a time dependent run with one vortex of aspect ratio $1: 1.5$. The time evolution of the system will be studied for much more than 1 tectonic cycle (overturn), so initial conditions do not play any role in the interpretation of results. The numerical procedure was described in detail in [Fradkov et al., 1995].

\section{Parameter Estimation}

Density number. For a first rough estimation of the density number $R_{p}=\frac{\beta C_{\circ}}{\alpha \Delta T}$, we use: $\alpha=1.4$. $10^{-5}{ }^{\circ} \mathrm{K}^{-1}$ [Jarvis and Peltier, 1985]; $\Delta T \approx 2000^{\circ} \mathrm{K}$; $\varrho_{f}=7.2 \frac{\mathrm{g}}{\mathrm{cm}^{3}}\left[\right.$ Ringwood, 1984]; $\varrho_{s}=4.1 \frac{\mathrm{g}}{\mathrm{cm}^{3}} ; C_{o}$ is given by $C_{0} \geq 0.045$. This gives us the chemical ' expansivity' $\beta=\frac{e_{f}-Q_{s}}{\varrho_{s}} \approx 0.756$ and the density number is given by $R_{p} \geq 1.2$. This estimate shows that the late stage of core formation can be described within the Boussinesq approximation.

Segregation velocity. The integral formulation of the conservation law (1) leads to a relation between the segregation velocity $v_{s}$, the averaged concentration of heavy component $C(t)$ in the mantle, and the velocity of growing core radius $\dot{R}_{c}$. This is the case if the integral formulation is applied to the shrinking mantle when considering the whole Earth as a closed (mass) system (e.g. Earth's radius $R_{E}$ is constant).

$$
\begin{array}{r}
\frac{d M_{c}}{d t}=4 \pi R_{c}^{2}(t) \varrho_{f} C(t)\left(v_{s}+\dot{R}_{c}\right) \\
\leftrightarrow C(t)=\frac{\dot{R}_{c}}{v_{s}+\dot{R}_{c}} \leftrightarrow \frac{d R_{c}(t)}{d t}=\frac{C(t)}{1-C(t)} v_{s}
\end{array}
$$

The differential equation governing $R_{c}(t)$ is obtained by differentiating the equation of the Earth's mass, which is composed of core mass and mantle mass,

$$
M_{E}=\frac{4}{3} \pi\left(\varrho_{f} R_{c}^{3}(t)+\left(C(t)\left(\varrho_{f}-\varrho_{s}\right)+\varrho_{s}\right)\left(R_{E}^{3}-R_{c}^{3}(t)\right)\right)
$$

and using the relation (3) for $C(t)$. The resulting ODE for $R_{c}(t)$ is

$$
0=\frac{d R_{c}^{3}(t)}{d t}+\frac{d}{d t}\left(\frac{\dot{R}_{c}}{v_{\varepsilon}+\dot{R}_{c}}\left(R_{E}^{3}-R_{c}^{3}(t)\right)\right)
$$

Table 1

\begin{tabular}{cc}
\hline Initial Core Radius & Segregation velocity \\
\hline $3.25 \times 10^{6} \mathrm{~m}$ & $0.30 \mathrm{~cm} / \mathrm{y}$ \\
$3.30 \times 10^{6} \mathrm{~m}$ & $0.19 \mathrm{~cm} / \mathrm{y}$ \\
$3.35 \times 10^{6} \mathrm{~m}$ & $0.12 \mathrm{~cm} / \mathrm{y}$ \\
\hline
\end{tabular}

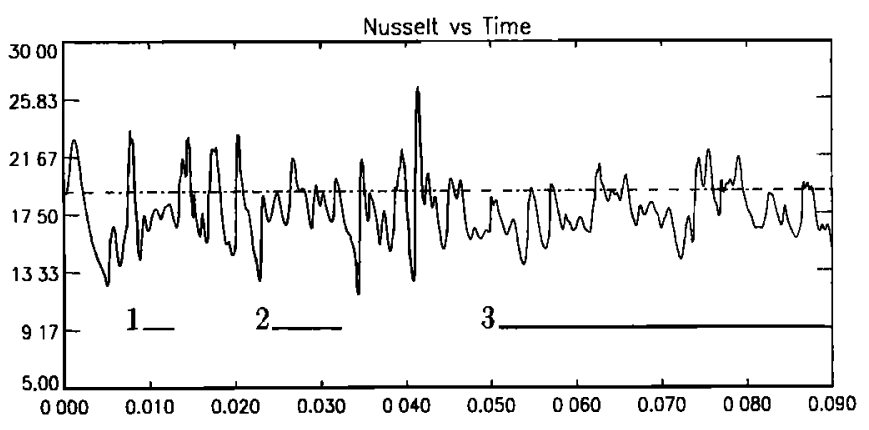

Figure 1. Nusselt vs time for the parameters $R_{T}=$ $10^{6}, R_{p}=1, a=20$. The dashed line shows the pure thermal convection case. Branch $1 \& 2$ indicate quasistable plume activity. Branch 3 shows a quasi-stable flow structure with one thermal plume from the top.

This equation can be solved analytically to give

$$
\begin{aligned}
t\left(R_{c}\right) & =\frac{R_{E}^{3}-a^{3}}{v_{s}}\left\{\frac{1}{a^{2} \sqrt{3}} \arctan \left(\frac{2 R_{c}+a}{a \sqrt{3}}\right)\right. \\
& \left.-\frac{1}{6 a^{2}} \ln \left(\frac{\left(a-R_{c}\right)^{2}}{a^{2}+a R_{c}+R_{c}^{2}}\right)\right\}+b
\end{aligned}
$$

where $a$ and $b$ are constants of integration. These are determined by the boundary conditions (core radius at the beginning and core radius at a later time). The additional constraint determining the separation velocity is given by the initial or final volume concentration $C$ of the 'iron' composition.

To get a conservative estimate, we stretch the time interval in which differentiation occurs to $3.0 \mathrm{~Gy}$. The final core radius is $3.48 \times 10^{6} \mathrm{~m}$ and the heavy material able to segregate into the core present in the initial state is $C\left(t_{i}\right)=0.045$. The estimates of $v_{s}$ for the initial core radius $R_{i}$ are listed in table 1.

\section{Numerical Results}

Figure 1 demonstrates the change in the time behavior of the Nusselt going from pure thermal convection (dashed line) to thermal convection with segregation (solid line), showing that the time behavior becomes irregular in the case of segregation. The time averaged Nusselt in figure 1 is smaller than for the pure thermal convection case, which is contrary to what was expected by Davies [1980] considering the thermal regime of the Earth during segregation. In figure 2 we present the corresponding temperature, concentration and stream function fields for selected time points. The first three panels of figure 2 show the development of a density-unstable temperature and concentration distribution near the lower boundary after the beginning of segregation. At the upper boundary, the FeO composition - carried under the top of the cell - has a density-stable distribution (positive buoyancy) and hinders the convection process (fig. 2.1; first minimum in $N u(t)$ ). The material thus stays longer at the horizontal boundaries, leading to over-cooling at the top (temperature unstable; concentration stable) and overheating plus differentiation (temperature and concentration unstable) at the bottom of the model domain, i.e. the segregation stabilizes the uppermost part of the cell and destabilizes the lower thermal boundary 


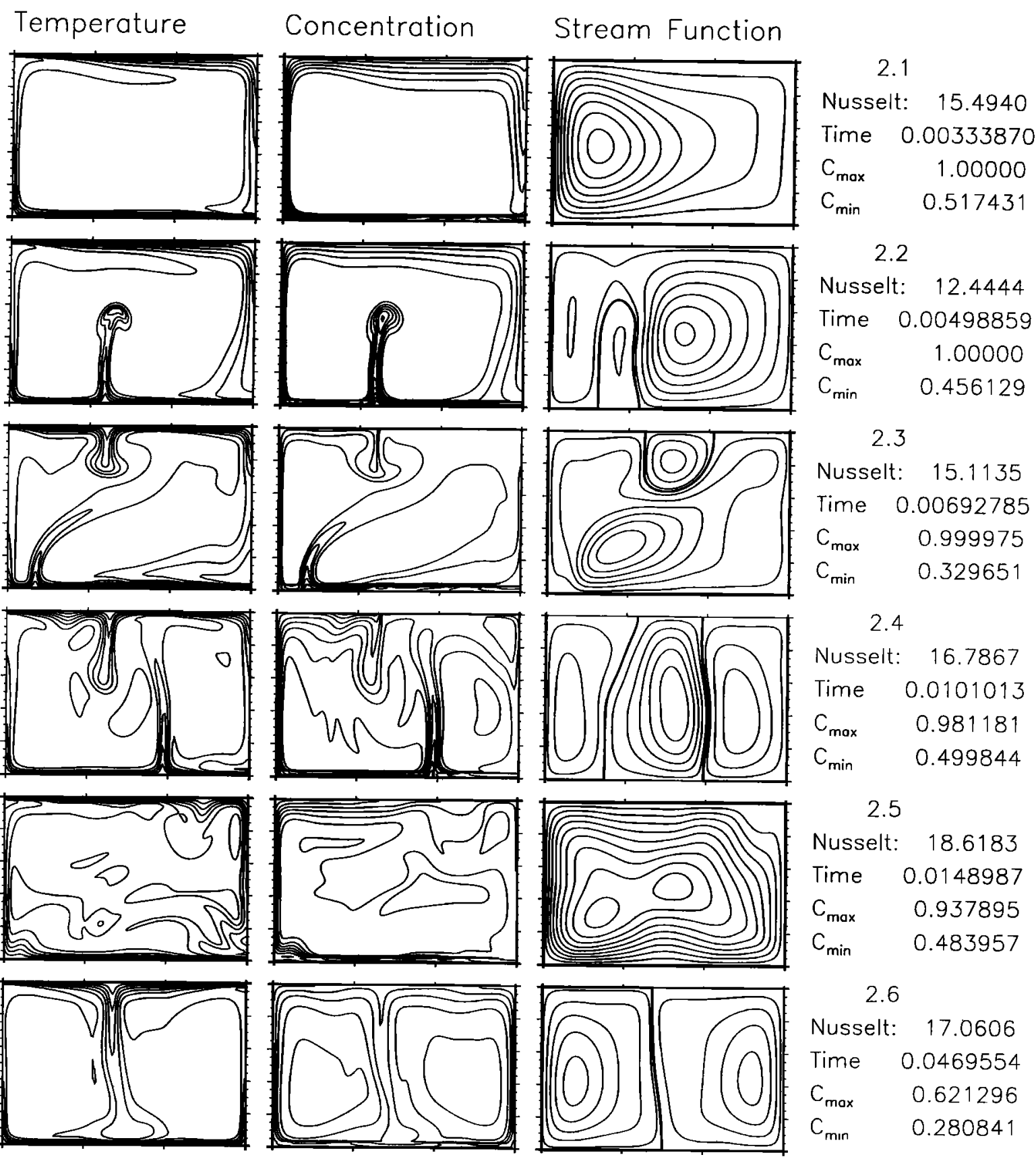

Figure 2. Contour plots of temperature, concentration and stream function in the different flow stages occurring during time evolution. Same parameters as in figure 1. Isocontours of concentration are equally spaced between $C_{\min }$ and $C_{\max }$. Nullisoline of stream-function is drawn thick. Panel 2.4 shows typical flow structure of quasisteady plume activity (Branch 1 \& 2 fig.1). Panel 2.5 shows the transition state (between Branch 1 \& 2 fig.1). Panel 2.6 show final flow stage which evolve to pure thermal convection case due to growths of one whirl.

layer. This density-instabilities are eased by the ascending (fig. 2.2, second maximum in $N u(t)$ ) and descending (fig. 2.3) of the plumes.

The above analysis of the temperature and concentration fields shows, that we can distinguish two kinds of plumes by their different causes: i) Up-welling plumes are driven by temperature and concentration (called thermo-compositional plumes (tcP)), ii) Down-welling plumes are driven only by temperature (called thermal plumes (tP)).
The analysis of the flow structure reveals that the system's evolution can be grouped into different kinds of stages, showed in the $N u(t)$ diagram.

The first stage (fig. 2.4; fig. 1 branches 1 \& 2) has a relatively steady flow structure on whole cell scale with a stably localized tcP plus tP and small amplitude oscillations of the $N u(t)$. The time duration is $\Delta t \approx$ $4.910^{-3}$ for branch 1 and $\Delta t \approx 11.210^{-3}$ for branch 2 .

The second stage, the so called transition state, (fig. 2.5 ; fig. 1 , between branches $1 \& 2$ and between 
branches 2 \& 3) has greater amplitude oscillations in the Nusselt as compared to branch 1 or 2 . The flow structure, extending over the whole cell, leads to the destruction of the plume structure.

Finally, we make some general remarks on the role of the density number and segregation parameter.

The density number has an important influence on system behavior in two ways: i) The density number triggers the birth of tcP's and stabilizes the localization of the tcP's, because $R_{p}$ together with the segregation parameter $a$ defines the magnitude of the 'chemicalbuoyancy production' at the lower boundary. ii) The ascend of tcP's is facilitated by the additional chemical buoyancy of the depleted material. This gives the tcP's a greater power to punch through the whole cell. The ability of a tcP to penetrate into the upper mantle depends on the density change in $670 \mathrm{~km}$ [Kellogg, 1991] and is not estimated quantitatively here. Contrary to the findings of Liu and Chase [1991], the chemical buoyancy persists as an important parameter for the ascend of the up-welling plume even for the 'infinite Lewis number' presented here because the plume source has a finite thickness in our case so that the thickness of the chemical plume stays finite in the limit $L e \rightarrow \infty$.

An increase of the segregation parameter shortens the time duration where the segregation process influences convective behavior of the mantle. This duration is not a simple linear function of the segregation parameter due to the nonlinearity of the problem. The effectiveness of mass removal of ' $\mathrm{FeO}$ ' depends also on the 'refreshing rate' of the lower layer where segregation occurs. This is a function of the overlying flow structure.

\section{Summary and Conclusions}

The main features of convection with segregation in the lower boundary are:

- Segregation of a heavy component in D" leads to a new kind of boundary layer instability. For that reason, the segregation process can strongly influence the convective behavior in the whole cell even if the additional mass transport due to segregation is small.

- Segregation destabilizes the lower thermal boundary layer and stabilizes the uppermost part of the cell.

- The flow structure shows two different kinds of plumes, distinguished by their various causes:

- plumes from the top are due to over-cooling (i.e. pure thermal origin)

- plumes from the bottom resulting from both over-heating and segregation (i.e. thermal \& compositional origin).

- The temporary behavior of the flow structure and the Nusselt can pass through different stages during the time evolution of the system:

- relatively steady small scale convection with small amplitude oscillation of the Nusselt; this flow structure has stably localized thermo-compositional plumes

- strong time-dependent convection with one whirl and great amplitude oscillation in the Nusselt.

The dynamics of mantle convection with segregation shows new traits for the Rayleigh number and geometry under consideration. We can conclude that this process can have structure dominating influence on mantle dynamics during Earth's history.

Acknowledgments. G.N. and A.S.F. would like to thank J. Arkani-Hamed (McGill-University Canada) for helpful discussions about geophysical applications of results. We would like to thank two anonymous reviewers and Mian Liu (University of Missouri) for their constructive reviews. We are grateful to D.J. Stevenson (Caltech) whose criticism removed an error in the early version of the paper. A.S.F. thanks the DFG (Germany) and NCERC (Canada) for financial support during the progression of this work.

\section{References}

Artyushkov, E.V., Density differentiation of the Earth's Matter and Processes at the Core-Mantle Interface, $J$. Geophys. Res., 77, 6454-6458, 1972

Boehler, R., Melting of the $\mathrm{Fe}-\mathrm{FeO}$ and the $\mathrm{Fe}-\mathrm{FeS}$ systems at high pressure: Constraints on core temperatures, Earth and Planet. Sci. Lett., 111, 217-227, 1992

Davies, G.F., Exploratore Models of the Earth's Thermal Regime During Segregation of the Core, J. Geophys. Res., $85,7108-7114,1980$

Fradkov, A.S., Nauheimer, G., Neugebauer, H.J., Volume separation in double-diffusive convection systems, in Double Diffusive Convection edited by A. Brand, H.J.S. Fernando, pp. 151-169, Geophys. Mono. vol. 94, 1995

Jarvis, G.T., Peltier, W.R., Convection models and geophysical observation, in Mantle Convection: Plate Tectonics and Global Dynamics edited by W.R. Peltier, pp. 479593, Gordon \& Breach N.Y., 1985

Kellogg, L.H., Interaction of plumes with a compositional boundary at $670 \mathrm{~km}$, Geophys. Res. Lett., 18, 865-868, 1991

Knittle, E., Jeanloz, R., Simulating the core-mantle boundary: An experimental study of high-pressure reactions between silicates and liquid iron, Geophys. Res. Lett., $16,609-612,1989$

Liu, Mian, Chase, Clement G., Boundary-layer model of mantle plumes with thermal and chemical diffusion and buoyancy, Geophys. J. Int., 104, 433-440, 1991

McCammon, C.A., Ringwood, A.E., Jackson, I., Thermodynamics of the system $\mathrm{Fe}-\mathrm{FeO}-\mathrm{MgO}$ at high pressure and temperature and a model for formation of the Earth's core, Geophys. J. R. astr. Soc., 72, 577-595, 1983

Oxburgh, E.R., O'Nions R.K., Helium loss, tectonics and the terrestrial heat budget, Science, 237, 1583-1588, 1987

Ringwood, A.E., The Earth's core: Its composition, formation and bearing upon the origin of the Earth, Proc. $R$. Soc. London A, 395, 1-46, 1984

Ringwood, A.E., Hibberson, W., Solubilities of mantle oxides in molten iron at high pressures and temperatures: implications for the composition and formation of Earth's core, Earth and Planet. Sci. Lett., 102, 235-251, 1991

Stevenson, D.J., Fluid dynamics of core formation, in Origin of the Earth edited by W.R. Peltier, pp. 231-249, Lunar Planet. Inst. Houston, 1991

Trubitzyn, V.P., Kharybin, E.V., Convective instability of the sedimentation state in the mantle, Physics of the solids Earth (Fizika zemli), N8 21-30, 1987

Vacquier, V., The origin of terrestical heat flow, Geophys. J. Int., 106, 199-202, 1991

G. Nauheimer, H.J. Neugebauer, Geodynamik, Nußallee 8. 53225 Bonn, GERMANY.(nauheim@geo.uni-bonn.de) A.S. Fradkov, Im Defdahl 51, 44141 Dortmund, GERMANY.(fradkov@prodv-gmbh.de)

(Received October 12, 1995; revised March 4, 1996; accepted May 16, 1996.) 